\title{
La résolution de problèmes en mathématiques au Québec : évolution des rôles assignés par les programmes et des conseils donnés aux enseignants \\ Solving math problems in Québec - changing roles assigned by programs and advice given to teachers \\ La resolución de problemas en matemáticas en Quebec: evolución de los roles asignados por los programas y los consejos dados a los maestros
}

Caroline Lajoie et Nadine Bednarz

Volume 42, numéro 2, automne 2014

Résolution de problèmes en mathématiques : un outil pour enseigner et un objet d'apprentissage

URI : https://id.erudit.org/iderudit/1027903ar

DOI : https://doi.org/10.7202/1027903ar

Aller au sommaire du numéro

Éditeur(s)

Association canadienne d'éducation de langue française

ISSN

1916-8659 (numérique)

Découvrir la revue

Citer cet article

Lajoie, C. \& Bednarz, N. (2014). La résolution de problèmes en mathématiques au Québec : évolution des rôles assignés par les programmes et des conseils donnés aux enseignants. Éducation et francophonie, 42(2), 7-23.

https://doi.org/10.7202/1027903ar
Résumé de l'article

La résolution de problèmes est un enjeu clé de l'enseignement des mathématiques à travers le monde et elle occupe une place importante dans les curricula. Pourtant, on a accordé peu d'attention aux défis que pose la résolution de problèmes dans l'enseignement. Ce questionnement nous a conduites à nous intéresser plus spécifiquement, dans le cas du Québec, au cadre référentiel fourni à l'enseignant pour aborder la résolution de problèmes avec les élèves. Que sait-on des conseils donnés aux enseignants pour aborder cette résolution en classe? Quelle cohérence entre ces conseils donnés aux enseignants et les rôles assignés à la résolution de problèmes dans l'enseignement? L'analyse cible plus spécifiquement les années 2000 au regard de ce qui a été déjà dégagé des périodes précédentes.
Tous droits réservés (C) Association canadienne d'éducation de langue française, 2014
Cecument est protégé par la loi sur le droit d'auteur. L'utilisation des services d'Érudit (y compris la reproduction) est assujettie à sa politique d'utilisation que vous pouvez consulter en ligne. 


\section{La résolution de problèmes en mathématiques au Québec: évolution des rôles assignés par les programmes et des conseils donnés aux enseignants}

\section{Caroline LAJOIE}

Université du Québec à Montréal, Québec, Canada

\section{Nadine BEDNARZ}

Université du Québec à Montréal, Québec, Canada

\section{RÉSUMÉ}

La résolution de problèmes est un enjeu clé de l'enseignement des mathématiques à travers le monde et elle occupe une place importante dans les curricula. Pourtant, on a accordé peu d'attention aux défis que pose la résolution de problèmes dans l'enseignement. Ce questionnement nous a conduites à nous intéresser plus spécifiquement, dans le cas du Québec, au cadre référentiel fourni à l'enseignant pour aborder la résolution de problèmes avec les élèves. Que sait-on des conseils donnés aux enseignants pour aborder cette résolution en classe? Quelle cohérence entre ces conseils donnés aux enseignants et les rôles assignés à la résolution de problèmes dans l'enseignement? L'analyse cible plus spécifiquement les années 2000 au regard de ce qui a été déjà dégagé des périodes précédentes. 


\section{ABSTRACT}

\section{Solving math problems in Québec - changing roles assigned by programs and advice given to teachers}

Caroline LAJOIE

University of Quebec in Montreal, Quebec, Canada

Nadine BEDNARZ

University of Quebec in Montreal, Quebec, Canada

Although problem solving is a key issue in mathematics teaching throughout the world and it holds an important place in the curriculum, little attention has been given to the challenges of problem solving in the context of teaching. This question led us to look more closely, in the case of Quebec, at the frame of reference provided to the teacher for approaching problem solving with students - what do we know about the advice given to teachers for dealing with this challenge in class? What consistency is there between the advice given to teachers and the roles assigned to problem solving? The analysis more specifically targets the 2000s in relation to what was already brought out in previous years.

\section{RESUMEN}

La resolución de problemas en matemáticas en Quebec: evolución de los roles asignados por los programas y los consejos dados a los maestros

Caroline LAJOIE

Universidad de Quebec en Montreal, Quebec, Canadá

Nadine BEDNARZ

Universidad de Quebec en Montreal, Quebec, Canadá

Si la resolución de problemas es un reto clave en la enseñanza de las matemáticas en todo el mundo y ocupa un lugar importante en los currículos, poca atención se ha dado a los retos que confronta la resolución de problemas en la enseñanza. Esta cuestión nos llevó a interesarnos más específicamente, en el caso de Quebec, al cuadro referencial que se le ofrece al maestro para abordar la resolución de problemas con sus alumnos: ¿Qué sabemos de los consejos que se dan a los maestro para abordar la resolución en clase? ¿Qué coherencia hay entre los consejos dados a los maestro y los roles asignados en la resolución de problemas? El análisis se orienta más específicamente hacia los años 2000 tomando en consideración lo que se conoce de los periodos precedentes. 


\section{Introduction}

Sur le plan international, plusieurs études se sont intéressées au développement de la résolution de problèmes dans les curricula (voir notamment Coppé et Houdement, 2009, pour la France; Artigue et Houdement, 2007, pour la France aussi; Santos-Trigo, 2007, pour le Mexique; Cai et Nie, 2007, pour la Chine; D’Ambrosio, 2007, pour le Brésil; Schoenfeld, 2007, pour les États-Unis; Hino, 2007, pour le Japon; ou encore Burkhardt et Bell, 2007, pour la Grande-Bretagne). Ces études ont contribué notamment à éclairer différentes composantes de la notion de problème et à dégager les multiples fonctions que joue la résolution de problèmes dans l'apprentissage et l'enseignement des mathématiques: appliquer des connaissances, permettre la construction de connaissances nouvelles, solliciter une activité de recherche mathématique, permettre l'utilisation partagée de plusieurs connaissances dans des situations complexes, travailler la modélisation, développer des heuristiques générales, etc. Ces travaux confirment que les problèmes ont constamment occupé une place dans les programmes et dans les instructions officielles. Ils permettent de comprendre comment la nature et les rôles dévolus à ces problèmes ont changé au fil du temps, en puisant à des influences multiples (Lajoie et Bednarz, 2012; Artigue et Houdement, 2007; Burkhardt et Bell, 2007; Schoenfeld, 2007). De l'énoncé des orientations données aux problèmes et de leur rôle dans l'enseignement, explicité dans ces travaux, à la pratique de cette résolution de problèmes, pensée dans un cadre d'enseignement, le saut est toutefois grand. Comme le font remarquer Burkhard et Bell (2007), peu d'attention semble avoir été accordée aux défis que ces approches peuvent poser pour les enseignants: «Failures in introducing novel elements into the implemented curriculum largely arise from the challenge being underestimated» (p. 403). Il ne suffit pas en effet de présenter des problèmes, d'expliciter différents critères de choix de ces problèmes, de préciser leurs rôles pour que les enseignants mettent en œuvre les fonctions dévolues à ces problèmes ou encore les processus complexes de résolution décrits dans ces programmes. Quel éclairage est fourni aux enseignants pour aborder la résolution de problèmes avec les élèves? Un tel éclairage est-il présent dans les documents institutionnels?

Cette préoccupation à l'égard des enseignants et de la résolution de problèmes nous a amenées à nous intéresser de plus près, pour le Québec, au cadre institutionnel pouvant guider leur pratique, leur manière d'approcher la résolution de problèmes en classe.

Peu de travaux ayant analysé les curricula se sont en effet intéressés à cette dimension ${ }^{1}$. Or, différentes recherches menées en collaboration avec des professionnels montrent l'importance de ce cadre institutionnel, qui agit à la fois comme contrainte mais aussi comme levier pour leur pratique. Comme nous le montrent par exemple les études de Lyet et Loreau (sous presse) et Roditi (2013), ce cadre est aussi partie prenante d'un espace d'assurance et d'ajustements de cette pratique, avec une

1. Cette expérience en classe est davantage au cœur des travaux menés au Japon (Hino, 2007) ou en Chine (Cai et Nie, 2007). Nous y reviendrons dans la discussion. 
dimension structurante de celle-ci. Il nous apparaît essentiel de regarder cette dimension institutionnelle pour mieux comprendre les défis que posera éventuellement la résolution de problèmes pour ces enseignants.

Cette étude vise ainsi à cerner ce cadre institutionnel à travers, d'une part, les conseils donnés aux enseignants qui sont susceptibles de guider leur manière d'aborder la résolution de problèmes en classe, et d'autre part, les liens, s'ils existent, entre ces conseils et les rôles assignés aux problèmes. En nous attardant à ces conseils au regard des intentions visées par la résolution de problèmes, nous mettons en évidence un élément clé du cadre référentiel fourni à l'enseignant pour approcher cette résolution.

\section{Quelques repères méthodologiques}

Le Québec est pris ici comme un «beau cas», au sens d'un «observatoire ${ }^{2}$ » privilégié dans lequel un certain objet peut être étudié (Hamel, 1997). La résolution de problèmes a en effet de tout temps été un élément important de la réalité de l'enseignement des mathématiques au Québec (Lajoie et Bednarz, 2012, sous presse). Elle a été un sujet constant de préoccupations de la communauté professionnelle en enseignement des mathématiques, plus particulièrement au primaire. Le Fascicule K (MEQ, 1988), un guide sur la résolution de problèmes qui s'adresse aux enseignants du primaire, témoigne déjà de l'importance du travail en ce domaine, mais on peut penser également aux rubriques régulières dont elle a fait l'objet dans la revue Instantanés mathématiques, s'adressant aussi aux enseignants du primaire ${ }^{3}$. Témoin aussi de cette effervescence au sujet de la résolution de problèmes, la mise sur pied par des membres de l'APAME (Association pour l'avancement des mathématiques à l'élémentaire) du concours Mathématlon autour duquel de nombreux problèmes seront expérimentés dans les écoles. Elle a été, par ailleurs, un sujet important de réflexions de la communauté didactique, comme en témoignent plusieurs publications sur ce sujet (voir notamment Lukenbein, 1984-1985; Lemoyne, Giroux et Biron, 1990; Lemoyne et Tremblay, 1986; Bednarz, 1996; Pallascio, 2005; Voyer, 2006; Mary et Theis, 2007). En ce sens, le Québec constitue un observatoire privilégié pour comprendre comment un certain objet lié à l'enseignement des mathématiques, un cadre référentiel fourni aux enseignants pour aborder la résolution de problèmes, s'est développé sur une longue période.

Signalons d'emblée que notre étude ne se situe pas sur le plan de la pratique effective en classe de la résolution de problèmes, mais bien sur le plan du cadre institutionnel pouvant éclairer l'action des enseignants dans ce domaine. Un ensemble

2. Le cas peut être défini comme un observatoire propre à cerner un objet (Hamel, 1997, p. 91). Ce n'est pas un lieu physique ou un contexte, mais bien une entité définie, un système délimité et un point d'observation pertinent pour notre objet d'étude.

3. Voir notamment Grignon, 1983; Landry, 1984-1985; Laforest, 1984-1985. Voir également «Une invitation au problème ouvert», une rubrique qui s'échelonne sur plusieurs numéros de 1996 et 1997; "Ouvertures pour un problème fermé », rubrique parue en 1998 (vol. 34, n² 2); «À partir d'un problème» une rubrique qui s'échelonne durant les années 1998 et 1999; et plus récemment «Les situations-problèmes: au cœur de la réforme en mathématiques» (Corbeil, Pelletier et Pallascio, 2001). 
de documents, produits au Québec et pour le Québec, permet de dégager ce cadre institutionnel:

- Les différents programmes d'études élaborés au Québec, qui permettent de situer les orientations données globalement à l'enseignement des mathématiques à l'école, et en particulier à la résolution de problèmes ${ }^{4}$.

- Des documents pédagogiques s'adressant aux enseignants, dans lesquels sont en quelque sorte explicitées les idées sous-jacentes aux programmes à l'égard de cette résolution ${ }^{5}$.

- Une série d'articles parus dans des revues pédagogiques, qui constituent une autre source importante de données pour capter les réflexions à l'égard de cette résolution ${ }^{6}$.

Cinq périodes, qui constituent des jalons importants de l'évolution des programmes, ont été considérées: le Québec d'avant la Seconde Guerre mondiale, celui de l'après-guerre, de l'après-Révolution tranquille (1960-1970), des années 19801990 et de la période actuelle (de 2000 à aujourd'hui). Nous nous intéressons plus particulièrement, dans cet article, à la période actuelle pour bien mettre en évidence les éléments nouveaux qui ressortent de ce cadre référentiel, en comparaison des études déjà menées sur les autres périodes (Lajoie et Bednarz, 2012). Cette période récente marque en effet une rupture avec ce qui précède à travers l'introduction de la notion de situation-problème (SP) et du critère de complexité qui la sous-tend (Lajoie et Bednarz, sous presse).

Avant d'entrer dans cette analyse, nous reviendrons brièvement sur les rôles dévolus à la résolution de problèmes (de situations-problèmes) dans l'enseignement, de manière à dégager les aspects les plus marquants de cette évolution. Celle-ci nous permettra de revenir par la suite sur la cohérence entre ces rôles et les conseils donnés aux enseignants (ces rôles agissant en quelque sorte comme fondement en arrière-plan de leur exploitation).

4. Dans les textes des programmes d'études, il est important de spécifier qu'aucune rubrique spécifique n'est consacrée aux rôles associés à la résolution de problèmes ni aux conseils donnés aux enseignants. Les programmes des années 2000 (MEQ, 2001, MELS, 2003, 2005), sur lesquels se centre principalement cet article, avec les documents associés, sont par exemple rédigés de la manière suivante: présentation générale de la discipline, contribution de la mathématique à l'apprentissage de l'élève, relations entre le programme de mathématiques et les autres éléments du programme de formation (domaines généraux de formation, compétences transversales, autres disciplines), contexte pédagogique, présentation de chacune des compétences, puis du contenu de formation (arithmétique, algèbre, géométrie, mesure, statistiques et probabilités). La présentation plus spécifique de chacune des compétences est structurée de la façon suivante: sens de la compétence, composantes, critères d'évaluation et attentes de fin de cycle. La section "contenu de formation" reprend le contenu spécifique, quelques éléments de méthode et des repères culturels. Ainsi, à des fins d'analyse, nous avons dû parcourir l'ensemble des documents, et ce, sous toutes les thématiques, de manière à identifier ce qui relevait des fonctions associées à la résolution de situations-problèmes ou des conseils donnés aux enseignants pour approcher cette résolution.

5. Voir Prototypes d'épreuves, MELS (2008).

6. Voir Corbeil et al. (2001) et Pallascio (2005). 


\section{Un bref retour sur les rôles assignés à la résolution de problèmes dans I'enseignement des mathématiques}

Notre analyse des documents institutionnels fait ressortir différentes fonctions assignées à la résolution de problèmes au cours du XXe siècle (Lajoie et Bednarz, 2012). Parmi ces fonctions (voir le tableau 1), deux ont été constamment présentes: une fonction d'application, traditionnellement réservée aux problèmes, mais aussi, même si cette dernière prend une place de moindre importance à certaines périodes, une fonction culturelle, de formation. Il y a là une première dualité centrale, caractéristique de ce que soulignait Gispert (2002) dans sa mise en perspective historique de l'enseignement des mathématiques en France.

Au-delà de ce premier constat, l'analyse plus fine des significations associées à chacune de ces fonctions fait apparaître des évolutions: de l'idée d'application vue comme une mise en pratique des acquis à celle de réinvestissement des concepts ${ }^{7}$; des problèmes forçant l'analyse et le raisonnement des notions aux problèmes visant le développement de la pensée mathématique et d'habiletés générales (généraliser, abstraire, etc.).

Par ailleurs, de nouvelles fonctions font leur apparition dès les années 1970 (voir le tableau 1), marquant une rupture avec ce qui précède. De problèmes avant tout attachés à une fonction d'application des connaissances, dans la première moitié du $\mathrm{XX}^{\mathrm{e}}$ siècle, et de ce fait souvent placés en fin de séquence d'enseignement, on passe, dès les années 1970, au recours à des problèmes dont l'intention est d'introduire, d'explorer de nouveaux concepts et propriétés mathématiques, un rôle qui va s'affirmer encore davantage dans les années 1980-1990. Désormais placés en début de séquence d'enseignement, ou au cours de celle-ci, les problèmes sont à la source de la construction de connaissances nouvelles. On observe enfin, au cours de cette évolution, un glissement plus contemporain du rôle des problèmes comme moyen d'étude, comme modalité pédagogique appuyant le travail en classe, à celui qu'ils remplissent comme objet d'étude. Ce nouvel enjeu s'accompagne d'un regard méta sur cette résolution. On cherche à développer par (dans) la résolution de problèmes des stratégies générales de résolution et des attitudes positives.

Ces différentes fonctions sont reprises avec l'introduction de la notion de situation-problème (voir le tableau 1). Ce qui marque toutefois ce début du XXI ${ }^{\mathrm{e}}$ siècle, c'est l'accent mis non plus uniquement sur les connaissances, mais aussi sur les processus mathématiques. Une telle présence peut en effet être repérée dans chacune des fonctions remplies par la résolution de situations-problèmes.

- Dans la fonction d'application: «le recours régulier à la résolution de situationsproblèmes en tant que modalité pédagogique permet à l'élève [...] d'élargir, d'approfondir et d'intégrer des concepts et des processus mathématiques» (MELS, 2003, p. 237).

7. Ainsi, si l'on parle encore d'application de connaissances dans les années 1980-1990, on le fait en des termes très différents, et le sens que prend cette application est dès lors autre. L'intention n'est plus seulement celle d'une utilisation de connaissances introduites au préalable, d'une mise en pratique de celles-ci. Elle est beaucoup plus ambitieuse: le problème est une occasion de revoir ces connaissances, d'en élargir le sens, de les approfondir, voire de faire des liens, d'intégrer des concepts. 
- Dans la fonction d'amorce à l'apprentissage: la résolution de situations-problèmes «permet à l'élève d'explorer, d'inventer, de construire [...] des concepts et des processus mathématiques» (MELS, 2003, p. 237).

- Dans sa fonction de formation: « [...] sur un plan plus abstrait, elle [la résolution de SP] s'avère aussi un outil intellectuel puissant au service du raisonnement et de l'intuition créatrice» (MEQ, 2001, p. 126).

C'est ce même processus qui est visé lorsqu'on aborde les SP comme objet d'apprentissage: «En tant que processus, la résolution de situations-problèmes constitue un objet d'apprentissage en soi» (MEQ, 2001, p. 124; MELS, 2003, p. 231; MELS, 2005, p. 1).

Tableau 1. Évolution de 1900 à nos jours des rôles associés aux problèmes (SP)

\begin{tabular}{|c|c|c|c|c|c|c|}
\hline & & 1904-1945 & 1946-1959 & $1960-1970$ & $1980-1990$ & $2000 \ldots$ \\
\hline \multirow{6}{*}{ 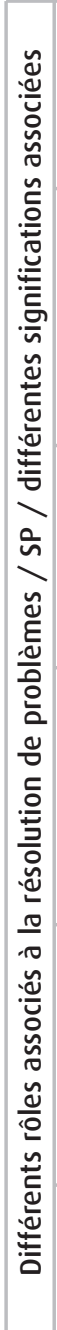 } & $\begin{array}{l}\text { Fonction } \\
\text { d'application }\end{array}$ & $\begin{array}{l}\text { Une fonction d'application } \\
\text { fortement liée au monde } \\
\text { de la pratique / une inten- } \\
\text { tion de mise en pratique } \\
\text { des connaissances }\end{array}$ & Idem & $\begin{array}{l}\text { Une intention } \\
\text { d'utilisation des } \\
\text { connaissances et } \\
\text { des techniques } \\
\text { apprises }\end{array}$ & $\begin{array}{l}\text { Une intention de } \\
\text { réinvestissement des } \\
\text { connaissances }\end{array}$ & $\begin{array}{l}\text { Une intention de } \\
\text { réinvestissement } \\
\text { des concepts et } \\
\text { des processus } \\
\text { mathématiques }\end{array}$ \\
\hline & $\begin{array}{l}\text { Fonction } \\
\text { de formation }\end{array}$ & $\begin{array}{l}\text { Une occasion de raisonner } \\
\text { les notions }\end{array}$ & Idem & $\begin{array}{l}\text { Une occasion } \\
\text { de développer } \\
\text { la pensée } \\
\text { mathématique }\end{array}$ & $\begin{array}{l}\text { Une occasion } \\
\text { de développer des } \\
\text { habiletés générales } \\
\text { (estimer, généraliser, } \\
\text { abstraire, etc.) }\end{array}$ & $\begin{array}{l}\text { Une occasion de } \\
\text { développer des } \\
\text { habiletés intel- } \\
\text { lectuelles faisant } \\
\text { appel au raison- } \\
\text { nement et à l'in- } \\
\text { tuition créatrice }\end{array}$ \\
\hline & $\begin{array}{l}\text { Fonction de } \\
\text { construction de } \\
\text { connaissances }\end{array}$ & & & $\begin{array}{l}\text { Un problème } \\
\text { comme amorce } \\
\text { à l'apprentis- } \\
\text { sage de } \\
\text { concepts, de } \\
\text { propriétés }\end{array}$ & $\begin{array}{l}\text { Un problème à la } \\
\text { source de la construc- } \\
\text { tion de connaissances } \\
\text { nouvelles }\end{array}$ & $\begin{array}{l}\text { Une SP qui permet } \\
\text { d'explorer, d'inven- } \\
\text { ter, de construire } \\
\text { des concepts et } \\
\text { des processus } \\
\text { mathématiques }\end{array}$ \\
\hline & $\begin{array}{l}\text { La résolution } \\
\text { de problèmes } \\
\text { comme objet } \\
\text { d'apprentissage }\end{array}$ & & & & $\begin{array}{l}\text { La résolution comme } \\
\text { objet d'étude et } \\
\text { habileté de base à } \\
\text { développer } \\
\text { (s'accompagne d'un } \\
\text { regard méta sur cette } \\
\text { résolution) }\end{array}$ & $\begin{array}{l}\text { Le processus de } \\
\text { résolution de SP } \\
\text { comme objet } \\
\text { d'apprentissage } \\
\text { (s'accompagne } \\
\text { d'un regard méta } \\
\text { sur ce processus) }\end{array}$ \\
\hline & $\begin{array}{l}\text { La résolution } \\
\text { de problèmes } \\
\text { comme } \\
\text { modalité } \\
\text { pédagogique }\end{array}$ & & & & $\begin{array}{l}\text { La résolution de } \\
\text { problèmes vue } \\
\text { comme une approche } \\
\text { pédagogique, un } \\
\text { moyen à privilégier } \\
\text { dans l'enseignement } \\
\text { des maths }\end{array}$ & $\begin{array}{l}\text { La résolution de SP, } \\
\text { en tant que moda- } \\
\text { lité pédagogique, } \\
\text { supporte la grande } \\
\text { majorité des dé- } \\
\text { marches d'appren- } \\
\text { tissage en maths }\end{array}$ \\
\hline & $\begin{array}{l}\text { Une fonction } \\
\text { plus générale }\end{array}$ & & & $\begin{array}{l}\text { Situation } \\
\text { servant } \\
\text { d'amorce à } \\
\text { l'apprentissage }\end{array}$ & & $\begin{array}{l}\text { La résolution de } S P \\
\text { pour développer } \\
\text { les autres compé- } \\
\text { tences }\end{array}$ \\
\hline
\end{tabular}


Un deuxième élément marque cette entrée dans le XXI ${ }^{\mathrm{e}}$ siècle, caractérisé par l'apparition de rôles plus généraux, en apparence plus ambitieux aussi par leur ampleur: exercer et développer les deux autres compétences du programme, déployer un raisonnement mathématique et communiquer à l'aide du langage mathématique (MELS, 2003, p. 240); et favoriser le développement de l'ensemble des compétences transversales, plus particulièrement en sollicitant la pensée créatrice de l'élève, en l'incitant à traiter de l'information, à rechercher l'efficacité dans son travail, souvent collectif, et à développer des façons appropriées de communiquer (MEQ, 2001, p. 126).

Ce rôle plus général sera quelque peu précisé dans le programme du second cycle du secondaire, où l'on fera ressortir le lien spécifique avec des compétences d'ordre intellectuel ou d'ordre méthodologique, telles notamment: la compétence transversale à résoudre des problèmes qui "partage [avec la résolution de situationsproblèmes en mathématiques] plusieurs éléments de stratégies et cerne de façon analogue le questionnement et la réflexion» (MELS, 2005, p. 8); la pensée créatrice, lorsqu'en «cherchant à résoudre une situation-problème, [l'élève] envisage plusieurs solutions, explore divers modèles, pistes et stratégies, laisse émerger ses intuitions, accepte le risque et l'inconnu, joue avec les idées, met à l'essai différentes façons de faire, explore de nouvelles stratégies et exprime ses idées sous de nouvelles formes " (MELS, 2005, p. 8); ou encore l'acquisition de méthodes de travail efficaces, l'élève confronté à une situation-problème devant "structurer sa pensée et organiser sa démarche, [et au moment] de présenter sa démarche et d'expliciter son raisonnement, [devant] recourir à des registres de représentation sémiotique appropriés et respecter les règles de rédaction des modèles de présentation retenus» (p. 8).

Comment ces éléments nouveaux, caractérisant les années 2000, vont-ils se traduire dans le cadre référentiel institutionnel, à travers les conseils donnés aux enseignants pour approcher la résolution?

\section{Que conseille-t-on aux enseignants pour aborder la résolution de situations-problèmes dans l'enseignement? Quelles continuités et quels changements par rapport aux périodes précédentes?}

À ce stade-ci, deux remarques principales s'imposent. Tout d'abord, notre analyse des différents documents officiels en ce début de XXI ${ }^{\mathrm{e}}$ siècle (MEQ, 2001; MELS, 2003, 2005) met en évidence que les conseils aux enseignants au regard de la résolution de SP sont souvent formulés de manière indirecte. Dans les programmes du primaire et du début du secondaire (MEQ, 2001; MELS, 2003), par exemple, ces conseils sont difficiles à repérer, puisqu'ils ne sont pas formulés explicitement. Qui plus est, ils ne sont pas regroupés dans une même section. Ensuite, si on trouve dans le programme du $2^{\mathrm{e}}$ cycle du secondaire beaucoup de conseils explicites à l'enseignant (presque tous condensés dans la section «contexte pédagogique»), ceux-ci ne se limitent pas à la résolution de SP. En effet, les conseils qu'on y trouve concernent 
parfois la résolution de situations d'une manière générale (celles-ci englobant les situations d'application, les situations-problèmes et les autres activités), parfois aussi l'enseignement des mathématiques d'une manière encore plus générale. Nous les avons tout de même pris en compte pour notre analyse.

\section{Des conseils sur la complexité des SP, en rupture avec les périodes précédentes mais en cohérence avec le nouvel accent mis sur les processus mathématiques}

Parmi les caractéristiques venant baliser le choix des SP par l'enseignant, celle qui distingue probablement le plus la période s'amorçant avec le $\mathrm{XXI}^{\mathrm{e}}$ siècle des périodes précédentes renvoie à une idée nouvelle de complexité (voir la section Repères méthodologiques). Nous avons montré l'importance que revêt ce critère de complexité pour le choix des SP, ce qu'il recouvre et la rupture qu'il présente par rapport à ce qui se faisait jusqu'alors (Lajoie et Bednarz, sous presse). Il s'agit donc là d'un enjeu central auquel sont confrontés les enseignants. Or cette nouvelle caractéristique s'accompagne uniquement de deux conseils distincts pour l'enseignant.

Tout d'abord, l'enseignant est encouragé à privilégier des situations complexes, c'est-à-dire des situations qui mobilisent l'ensemble des composantes d'une compétence, représentent un défi intellectuel, suscitent un conflit cognitif, favorisent la prise de risques et se prêtent à plus d'une démarche (MELS, 2005, p. 14). Parmi les paramètres à considérer dans le choix (ou la construction) des SP, plusieurs caractérisent en fait la complexité des situations considérées: l'étendue des concepts et processus à mobiliser, le degré de familiarité/degré d'autonomie exigé de l'élève, le nombre de contraintes, les types de registres de représentation sollicités et les passages entre ces registres, le nombre d'étapes que comporte la solution, le niveau d'abstraction exigé pour s'approprier la situation, la nature des liens intra-mathématiques sollicités (concepts et processus, liens entre plusieurs champs des mathématiques) ou interdisciplinaires, etc.

Ensuite, des allers-retours sont suggérés entre des situations complexes et des situations simples en cours de processus d'apprentissage.

L'enseignant doit s'assurer que l'élève progresse dans le développement de ses compétences. Plusieurs paramètres balisant cette progression interviennent au regard de la complexité des situations d'apprentissage et d'évaluation [...] Ces paramètres n'évoluent pas nécessairement de façon linéaire. Des allers-retours sont donc souhaitables entre le simple et le complexe, entre le concret et l'abstrait [en référence aux objets ou aux contextes] ou entre le quantitatif et le qualitatif [pas de détails dans le programme sur ce à quoi ces termes réfèrent] (MELS, 2005, p. 15).

Ces deux conseils détonnent par rapport à d'autres donnés au cours de la première moitié du XXe siècle. En effet, cette idée de complexité des situations proposées prend la place d'un souci d'adaptation à l'enfant et celle d'alternance entre des situations simples et des situations complexes remplace une idée de gradation des problèmes du plus facile au plus difficile (pour de nombreux extraits de documents 
tirés de la première moitié du XX $\mathrm{X}^{\mathrm{e}}$ siècle, voir Lajoie et Bednarz, 2012). Ce conseil de privilégier des situations complexes est toutefois à rapprocher de l'accent mis non plus uniquement sur les connaissances, comme c'était le cas précédemment, mais aussi sur les processus mathématiques ainsi que sur le développement des autres compétences disciplinaires (raisonner, communiquer à l'aide du langage mathématique) (voir la première section). Il est donc cohérent avec les fonctions nouvelles dévolues aux SP faisant leur apparition en ce début de XXI ${ }^{\mathrm{e}}$ siècle.

\section{Des conseils liés au choix de contextes qui reflètent les nouveaux rôles} plus généraux assignés à la résolution de SP

Parmi les nouveaux critères pouvant guider le choix des SP par l'enseignant, on trouve dans les programmes des années 2000 des critères qui concernent un recours à des contextes inspirés des autres disciplines (MELS, 2003, p. 237; MELS, 2005, p. 9 et 10), des domaines généraux de formation (environnement, médias, entrepreneuriat, etc.), du contexte historique dans lequel a évolué la mathématique (MELS, 2003, p. 237), ou encore des contextes liés au marché du travail. Dans ce dernier cas, par exemple, on conseille explicitement à l'enseignant de proposer à l'élève «des situations d'apprentissage qui intègrent des contextes liés au marché du travail pour favoriser chez lui l'exploration professionnelle et aussi l'aider à mieux cerner ses goûts, ses champs d'intérêt et ses aptitudes particulières» (MELS, 2005, p. 15). Cette référence à des macro-contextes permet de rejoindre la fonction plus générale associée aux SP dans l'enseignement des mathématiques (voir la première section), ces macro-contextes sollicitant notamment des compétences d'ordre méthodologique d'organisation et de travail efficaces.

\section{À propos de la démarche de résolution: des conseils qui traversent les époques tout en laissant de plus en plus de latitude à l'enseignant}

À travers les différentes réformes, plusieurs conseils relatifs à la démarche de résolution de problèmes sont donnés à l'enseignant. Si ces conseils traversent essentiellement les époques, ils ne sont toutefois pas formulés de la même manière au fil du temps. Du début du XXe siècle jusqu'aux années 1970, quelques conseils précis sont formulés, lesquels mettent l'accent sur l'analyse du problème, le fait de «raisonner le problème», la visualisation du problème (se fermer les yeux, voir la scène), le support (au raisonnement) du dessin et de la manipulation, les traces de la démarche (Lajoie et Bednarz, 2012).

Dans les documents produits au cours des années 1980, on trouve un grand nombre de conseils à l'endroit de l'enseignant quant aux interventions à privilégier et aux stratégies à mettre en place, mais ces conseils, quoique explicites, sont déjà beaucoup plus globaux, moins spécifiques, et ce, dans un but avoué de «laisser une grande liberté d'action à l'enseignante ou à l'enseignant quant aux façons de les intégrer dans leur pratique pédagogique» (MEQ, 1993, p. 17). Comme nous l'avons fait remarquer ailleurs:

[...] cette évolution des conseils donnés aux enseignants, beaucoup plus spécifiques de 1900 à 1960, plus généraux dans les années 80, est sans 
doute à examiner en parallèle avec l'évolution de la conception du rôle de l'enseignant (Laplante, 1984-1985). Le mouvement de professionnalisation de l'enseignement se fait en effet sentir dès les années 80 à travers la manière dont on conçoit son intervention en résolution de problèmes: «Le rôle de l'enseignant dans la démarche de résolution de problème est alors plus complexe parce que plus effacé. La formation de base que l'on souhaite donner à l'apprenant est moins orientée vers la technique mais davantage intégrée au développement global de l'enfant. Il s'agit pour l'enseignant de s'impliquer lui-même dans une démarche plutôt que d'être un démonstrateur de solutions. Les exigences de cette approche changent les règles du jeu pour l'enfant et pour l'enseignant. Cela concerne les attitudes, l'environnement de la classe, les situations-problèmes à identifier et, bien sûr, les interventions didactiques (Laplante, 1984-1985, p. 17)» (Lajoie et Bednarz (2012, p. 207-208)).

Dans les documents des années 2000, la tendance se poursuit, et les conseils à l'égard de la démarche de résolution de SP doivent être dégagés, et même déduits, d'une lecture approfondie du programme. En effet, l'enseignant doit déduire ce qui est attendu de lui à l'égard de la démarche de résolution de SP à partir de la description de ce qui est attendu de ses élèves, comme en témoigne l'extrait suivant (MELS, 2003, p. 241), qui consiste en le libellé de la compétence à résoudre des SP.

Figure 1. Compétence à résoudre des situations-problèmes (MELS, 2003, p. 241)

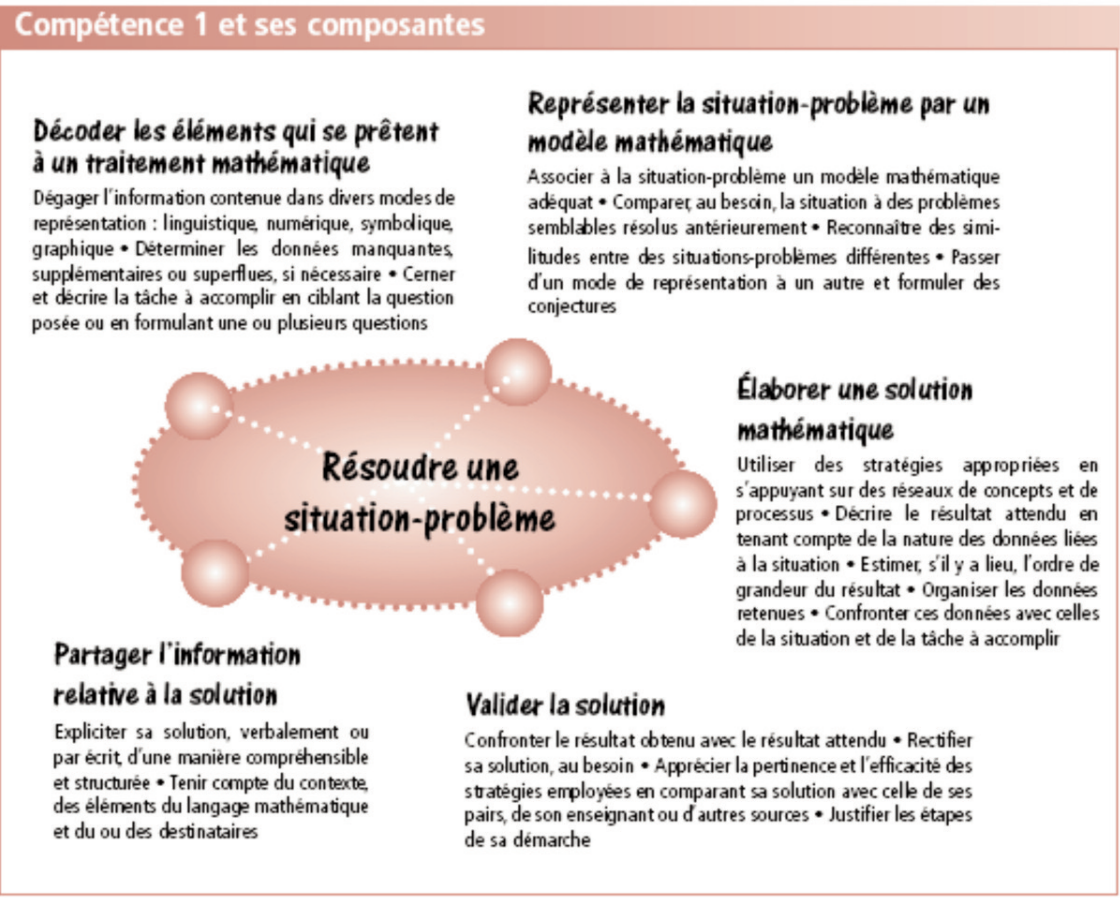


Aussi, des «exemples de stratégies associées à la résolution de situations-problèmes" sont fournis à l'enseignant, lui permettant par le fait même d'induire des éléments susceptibles de le guider dans sa manière de travailler la résolution de SP avec ses élèves.

Figure 2. Exemples de stratégies associées à la résolution de situations-problèmes et pouvant être développées par l'élève au moment de l'exercice de ses compétences

(MELS, 2003, p. 262)

\section{Compréhension}

- Distinguer les termes du langage courant et du langage mathématique

- Se représenter la situation mentalement ou par écrit

- Dégager la tâche à réaliser

- Reformuler la situation dans ses propres mots

Organisation

- Etablir des liens

- Mobiliser les concepts et les processus

- Utiliser des listes, des tableaux, des schémas, du matériel concret, des dessins

\section{Solution}

- Procéder par essais et erreurs

- Faire des retours sur son travail (travailler à rebours)

- Se référer à un problème analogue déjà résolu

- Diviser un problème complexe en sous-problèmes

- Simplifier le problème

Validation

- Vérifier sa solution à l'aide d'exemples ou par un raisonnement

- Utiliser d'autres processus, s'il y a lieu

- Chercher des contre-exemples

- Comparer et confronter ses démarches et ses résultats avec ceux de son enseignant ou de ses pairs

\section{Communication}

- Structurer ses idées

- Confronter sa compréhension de mots communs au langage courant et au langage mathématique

- Mobiliser différents modes de représentation

- Expérimenter différentes façons de transmettre un message à caractère mathématique

- Expliquer son raisonnement

On remarque, à la lecture de ces extraits, que non seulement les conseils à l'enseignant sont moins directs et plus généraux qu'ils ne l'étaient auparavant, mais qu'ils sont aussi plus «diffus», en ce sens qu'il faut les dégager d'une longue liste d'attentes à l'égard de la démarche de résolution par l'élève.

Il faut ajouter ici, en lien avec la démarche de résolution, un conseil adressé à l'enseignant, qui ne concerne pas que les SP mais qui est lié au développement des compétences, reprenant l'idée d'un retour métacognitif: «l'enseignant doit amener l'élève à prendre conscience, d'une part, de la façon dont il construit et mobilise ses 
savoirs dans diverses situations et, d'autre part, de la possibilité de les réutiliser dans d'autres situations en les adaptant au contexte» (MELS, 2005, p. 13).

\section{La différenciation pédagogique : un nouveau principe plus large derrière plusieurs conseils en continuité avec la ou les périodes précédentes}

Avec la réforme des années 2000, la variété des problèmes (SP), une des caractéristiques s'étant élargie au fil des différentes réformes (d'une variété des contextes dans un but avoué d'empêcher la monotonie et l'ennui on est passé à une variété au niveau des types de contextes, du nombre de solutions, de l'adéquation des données, des modes de représentations utilisés, dans un but plus implicite de varier les apprentissages) (Lajoie et Bednarz, 2012, p. 201), s'inscrit dorénavant dans un phénomène plus large de différenciation pédagogique visant à amener l'élève à développer au maximum sa culture mathématique (MELS, 2003, p. 237), de même que ses potentialités (MELS, 2005, p. 13).

Ainsi, en lien avec ce phénomène plus large de différenciation pédagogique, on trouve dans les programmes du primaire et du secondaire plusieurs pistes pour l'enseignant. Si ces pistes apparaissent implicitement dans le programme du primaire, elles apparaissent plus explicitement dans ceux du secondaire ${ }^{8}$, en particulier dans celui du deuxième cycle. Parmi les «pistes favorisant une pratique de différenciation" (MELS, 2005, p. 14), on trouve les suivantes, que nous avons pu relier en partie à certains conseils donnés aux enseignants au $\mathrm{XX}^{\mathrm{e}}$ siècle:

- amener les élèves à concevoir eux-mêmes des situations (un conseil qui traverse les époques);

- offrir aux élèves la possibilité de choisir entre des situations qui font appel aux mêmes concepts et processus, mais dont les contextes diffèrent (cette possibilité de choix de problèmes par les élèves parmi une liste de problèmes était déjà présente dans les années 1980-1990);

- proposer des situations d'apprentissage qui peuvent être exploitées dans différents champs de la mathématique ou à l'aide de différents registres de représentation sémiotique;

- varier les modalités d'organisation de la classe: activités individuelles ou de coopération (cette idée d'encourager le travail en équipe sans négliger le travail individuel est en continuité avec l'époque précédente).

8. Dans le programme du premier cycle du secondaire (MELS, 2003, p. 237), par exemple, il est mentionné que les situations peuvent être réalisées individuellement ou en équipe, en classe ou à la maison; que leur objet renvoie à des situations pratiques plus ou moins familières, réelles ou fictives, réalistes ou fantaisistes, ou encore purement mathématiques; qu'elles sont inspirées des autres disciplines, de l'environnement de l'élève, des domaines généraux de formation ou du contexte historique dans lequel a évolué la mathématique; qu'elles comportent des données complètes, superflues, implicites ou manquantes; qu'elles peuvent conduire à un ou plusieurs résultats ou, au contraire, ne mener nulle part. 


\section{Discussion}

Notre analyse a fait ressortir le caractère de plus en plus ambitieux des fonctions assignées à la résolution de situations-problèmes en ce début de XXI ${ }^{\mathrm{e}}$ siècle. Cellesci intègrent le développement de processus mathématiques et de compétences débordant du champ des mathématiques. Cela va se traduire par des conseils donnés aux enseignants, cohérents avec ces fonctions, privilégiant notamment le recours à des situations complexes, à des contextes issus d'autres disciplines, des domaines généraux de formation ou encore du monde du travail. Quant à l'exploitation en classe de ces situations, les conseils se révèlent ici très généraux, mettant de l'avant le recours aux interactions dans cette exploitation (travail en équipe, confrontation des solutions et points de vue), la nécessité d'un retour métacognitif ou encore la possibilité que les élèves choisissent les situations qui seront résolues, voire même qu'ils en formulent. Les éléments importants à travailler, et susceptibles d'orienter l'action des enseignants, restent implicites et doivent être déduits indirectement de ce qui est attendu des élèves. Ainsi, ce qui frappe au terme de cette analyse des années 2000, c'est la quasi-absence de conseils directs destinés à l'enseignant ainsi que le peu d'indications qui lui sont fournies pour approcher ces situations en classe. Le cadre référentiel institutionnel, tel qu'il se dégage de cette analyse, laisse ainsi beaucoup de latitude à l'enseignant dans le choix des SP et dans leur mise en œuvre en classe. Cette ouverture, qui s'inscrit dans le mouvement de professionnalisation de l'enseignement amorcé dans les années 1980-1990, peut s'avérer une arme à deux tranchants. Elle donne en effet lieu à de multiples interprétations possibles, laissant place à un terreau fertile, mais peut en même temps être une source importante d'insécurité pour les enseignants, notamment face aux défis que risque de poser la complexité de ces situations lorsqu'elles seront abordées avec les élèves en classe. Quels défis soulèvent l'analyse de ces situations par les élèves et leur appropriation? Quelles pistes permettent d'approcher cette analyse? Rappelons ici que l'analyse du problème était au cœur de plusieurs conseils précis donnés aux enseignants dans les années 1900-1960 (Lajoie et Bednarz, 2012). Elle semble ici peu prise en compte ou tout au moins sous-estimée. D'autres questions se posent également en lien avec cette complexité. Comment choisir la situation problème qui sera proposée aux élèves? Sur quelle base? Qu'est-ce qui en fait la complexité? De quelle nature est cette complexité? Comment gérer le processus de recherche des élèves à partir des questions et pistes qu'ils retiennent? Quelle synthèse peut-on envisager à partir d'un tel type de situation et de son exploitation? Autant de questions qui se posent à l'enseignant et pour lesquelles le cadre institutionnel apporte peu d'éléments. Or, comme le montraient bien Burkhard et Bell (2007), les défis que ces approches peuvent poser aux enseignants, et qu'on perçoit bien à travers ces questions, méritent qu'on s'y attarde. Ils montrent le travail qui reste à mener, en formation initiale et continue des enseignants, autour du pilotage de ces situations-problèmes en classe. Les travaux menés au Japon autour des «lesson studies» ouvrent ici des pistes intéressantes (Hino, 2007). De telles études, impliquant activement des enseignants, s'articulent sur des leçons (les leurs) construites autour de problèmes, des leçons que ces 
enseignants partagent, investiguent, interrogent à partir des vidéos en classe, modifient en essayant d'intégrer les points discutés. Ces réflexions permettent d'éclairer progressivement, pour ces enseignants, l'organisation possible d'une leçon construite autour d'un problème, le questionnement de l'enseignant à différentes phases, et ce en lien avec le développement de la pensée mathématique des élèves.

Sur le plan de la recherche, de tels travaux permettraient de mieux comprendre les manières de faire qui se constituent chez les enseignants pour approcher la résolution de situations-problèmes avec les élèves : les critères-en-acte qui guident la sélection, l'aménagement par des enseignants des situations retenues; les manières de faire autour de la présentation et de l'analyse de la situation avec les élèves; les manières dont est approchée la phase de recherche avec les élèves, ou les retours sur les solutions ainsi que la synthèse. Tout un champ de recherche à explorer pour mieux comprendre le travail de l'enseignant autour de ces situations et les défis qu'il pose!

\section{Références bibliographiques}

ARTIGUE, M. et HOUDEMENT, C. (2007). Problem solving in France: Didactic and curricula perspectives. Zentralblatt für Didaktik der Mathematik (ZDM), 39, 365-382.

BEDNARZ, N. (1996). Language activities, conceptualization and problem solving. The role played by verbalization in the development of mathematical thought by young children. Dans H. M. Mansfield, N. A. Pateman et N. Bednarz (dir.), Mathematics for Tomorrow's Young Children: International Perspectives on Curriculum (p. 228- 239). Dordrecht: Kluwer.

BEDNARZ, N. (2002). Pourquoi et pour qui enseigner les mathématiques? Une mise en perspective historique de l'évolution des programmes au Québec au XX ${ }^{\mathrm{e}}$ siècle. Zentralblatt für Didaktik der Mathematik (ZDM), 34(4), 146-157.

BURKHARDT, H. et BELL, A. (2007). Problem solving in the United Kingdom. Zentralblatt für Didaktik der Mathematik (ZDM), 39, 395-403.

CAI, J. et NIE, B. (2007). Problem solving in Chinese mathematics education: Research and practice. Zentralblatt für Didaktik der Mathematik (ZDM), 39, 459-473.

COPPÉ, S. et HOUDEMENT, C. (2009). Résolution de problèmes à l'école primaire française: perspectives curriculaire et didactique. Actes $d u 36^{e}$ colloque de la Commission permanente des IREM pour l'enseignement des mathématiques à l'école élémentaire (COPIRELEM).

CORBEIL, N., PELLETIER, M. et PALLASCIO, R. (2001). Les situations-problèmes: au cœur de la réforme en mathématiques. Instantanés mathématiques, 32(3), 12-27. 
D’AMBROSIO, U. (2007). Problem solving: A personal perspective from Brazil. Zentralblatt für Didaktik der Mathematik (ZDM), 39, 515-521.

GISPERT, H. (2002). Pourquoi, pour qui enseigner les mathématiques? Une mise en perspective historique de l'évolution des programmes de mathématiques dans la société française au XX ${ }^{\mathrm{e}}$ siècle. Zentralblatt für Didaktik der Mathematik (ZDM), 34(4), 158-163.

GRIGNON, J. (1983). La résolution de problèmes, à la recherche d'un nouveau modèle. Instantanés mathématiques, 19(3), 3-7.

HAMEL (1997). Étude de cas et sciences sociales. Paris : L'Harmattan.

HINO, K. (2007). Toward the problem-centered classroom: Trends in mathematical problem solving in Japan. Zentralblatt für Didaktik der Mathematik (ZDM), 39, 503-514

LAFOREST, J.-C. (1984-1985). L'évaluation des apprentissages en résolution de problèmes: une pratique essentielle et enrichissante. Instantanés mathématiques, 21 (numéro spécial D), 23-29.

LAJOIE, C. et BEDNARZ, N. (2012). Évolution de la résolution de problèmes en enseignement des mathématiques au Québec: un parcours sur cent ans des programmes et documents pédagogiques. Revue canadienne de l'enseignement des sciences, des mathématiques et des technologies, 12(2), 178-213.

LAJOIE, C. et BEDNARZ, N. (sous presse). La notion de situation-problème en mathématiques au début $\mathrm{du} 21^{\mathrm{e}}$ siècle au Québec: rupture ou continuité? Revue canadienne de l'enseignement des sciences, des mathématiques et des technologies.

LANDRY, Y. (1984-1985). Résolution de problèmes et processus créateur ou à la recherche d'une pratique créatrice. Instantanés mathématiques, 21 (numéro spécial C), 23-39.

LAPLANTE, H. (1984-1985). L'enseignant dans la démarche de résolution de problèmes. Instantanés mathématiques, 21 (numéro spécial D), 17-18.

LEMOYNE, G., GIROUX, J. et BIRON, D. (1990). Connaissances utilisées par des élèves de 8 à 12 ans dans la formulation de problèmes arithmétiques concrets. European Journal of Psychology of Education, 5(3), 273-291.

LEMOYNE, G. et TREMBLAY, C. (1986). Addition and multiplication: Problemsolving and interpretation of relevant data. Educational Studies in Mathematics, 17(2), 97-123.

LUKENBEIN, D. (1984-1985). La résolution de problèmes et le processus d'apprentissage en mathématique. Instantanés mathématiques, 21 (numéro spécial D), 5-9. 
LYET, P. et LOREAU, F. (sous presse). Effets en termes de légitimation des travailleurs sociaux et des chercheurs dans une recherche action collaborative sur le travail avec les parents en protection de l'enfance. Dans les actes du colloque pluridisciplinaire et plurisectoriel Les recherches-actions collaboratives: une révolution silencieuse de la connaissance. IRTESS de Bourgogne, France, 27-29 mai 2013.

MARY, C. et THEIS, L. (2007). Les élèves à risque dans des situations problèmes statistiques: stratégies de résolution et obstacles cognitifs. Revue des sciences de l'éducation, 33(3), 579-599.

MINISTÈRE DE L'ÉDUCATION DU QUÉBEC (MEQ) (1988). Guide pédagogique. Primaire. Mathématique. Résolution de problèmes • Orientation générale. Fascicule K (Document 16-2300-11). Québec: Gouvernement du Québec.

MINISTÈRE DE L'ÉDUCATION DU QUÉBEC (MEQ) (2001). Programme de formation de l'école québécoise. Version approuvée. Éducation préscolaire et enseignement primaire. Québec: Gouvernement du Québec.

MINISTÈRE DE L'ÉDUCATION, DU LOISIR ET DU SPORT (MELS) (2003). Programme de formation de l'école québécoise. Enseignement secondaire, premier cycle. Québec: Gouvernement du Québec.

MINISTÈRE DE L'ÉDUCATION, DU LOISIR ET DU SPORT (MELS) (2005). Programme de formation de l'école québécoise. Enseignement secondaire, deuxième cycle. Québec: Gouvernement du Québec.

PALLASCIO, R. (2005). Les situations-problèmes: un concept central du nouveau programme de mathématique. Vie pédagogique, 136, 32-36.

POIRIER PROULX, L. (1999). La résolution de problèmes en enseignement. Cadre référentiel et outils de formation. Paris: De Boeck et Larcier.

RODITI, E. (2013). Le métier d'enseignant et l'éclairage de la recherche collaborative. Dans N. Bednarz (dir.), Recherche collaborative et pratique enseignante (p. 350-363). Paris: L'Harmattan.

SANTOS-TRIGO, M. (2007). Mathematical problem solving: An evolving research practice domain. Zentralblatt für Didaktik der Mathematik (ZDM), 39, 523-536.

SCHOENFELD, A. H. (2007). Problem solving in the United States, 1970-2008: Research and theory, practice and politics. Zentralblatt für Didaktik der Mathematik (ZDM), 39, 537-551.

VOYER, D. (2006). L'influence de facteurs liés à l'élève ou à l'énoncé sur la compréhension en résolution de problèmes écrits d'arithmétique. Thèse de doctorat en éducation. Québec: Université Laval. 